\title{
Characteristics, incidence, and risk factors for death from fatal pneumonia among patients with primary malignant bone tumors: a SEER-based observational study
}

\author{
Kaixu Yu', Ying Chen ${ }^{2}$, Yahao Tian ${ }^{1}$, Honglei Kang', Kehan Song ${ }^{1}$, Yimin Dong ${ }^{1}$, Renpeng Peng ${ }^{1}$, \\ Feng $\mathrm{Li}^{1}$
}

${ }^{1}$ Department of Orthopedics, Tongji Hospital, Tongji Medical College, Huazhong University of Science and Technology, Wuhan, China; ${ }^{2}$ Department of Obstetrics and Gynecology, Tongji Hospital, Tongji Medical College, Huazhong University of Science and Technology, Wuhan, China

Contributions: (I) Conception and design: K Yu, F Li; (II) Administrative support: F Li; (III) Provision of study materials or patients: Y Chen, Y Tian; (IV) Collection and assembly of data: H Kang, K Song; (V) Data analysis and interpretation: K Yu, Y Dong, R Peng; (VI) Manuscript writing: All authors; (VII) Final approval of manuscript: All authors.

Correspondence to: Feng Li, PhD. Department of Orthopedics, Tongji Hospital, Tongji Medical College, Huazhong University of Science and Technology, Wuhan 430030, China. Email: lifengmd@hust.edu.cn.

Background: (I) To determine whether patients with malignant bone tumors had a higher risk of dying from pneumonia compared with the general US population; (II) to identify the independent risk factor associated with fatal pneumonia among these patients.

Methods: We identified 18,583 patients diagnosed with primary malignant bone tumors between 1973 and 2016 from the Surveillance, Epidemiology, and End Results (SEER) database. Standardized mortality ratios (SMRs) were calculated based on the mortality data of the general population gathered by the National Center for Health Statistics, which provided the risk of death from pneumonia among cancer patients relative to that of the general population. Given that other causes of death were considered as competing events, we also designed the Fine-Gray model to identify demographic and tumor-related characteristics associated with a higher risk of dying from pneumonia among these patients.

Results: Patients with primary malignant bone tumors had a higher risk of dying from pneumonia than the general population after adjusting the distribution difference of age, sex, and race among them (SMR =2.79; 95\% CI: 2.17-3.59). The older age, Black and earlier period of diagnosis were found to be the independent prognostic factor for a higher risk of death from pneumonia for these patients. Additionally, amputation due to malignant bone tumors significantly increased the risk of death from pneumonia compared with nonsurgery. The highest mortality rate of pneumonia was observed among patients with chordoma. Interaction tests demonstrated that amputation only increased the relative risk of fatal pneumonia among patients with osteosarcoma. Throughout the follow-up period, the mortality rate of fatal pneumonia was the highest within the first year after diagnosis, and the highest relative suicide risks persisted over time in patients with osteosarcoma.

Conclusions: To mitigate the risk of fatal pneumonia among patients with bone tumors, we call for longterm clinical monitoring of the lung condition among these patients, especially for those after amputation for bone tumors.

Keywords: Malignant bone tumors; pneumonia; standardized mortality ratio (SMR); amputation

Submitted Feb 18, 2021. Accepted for publication Jul 02, 2021.

doi: $10.21037 /$ tcr-21-306

View this article at: https://dx.doi.org/10.21037/tcr-21-306 


\section{Introduction}

Pneumonia and cancer are the leading causes of death worldwide (1). In 2017, it was estimated that more than 2.56 million people died from pneumonia and approximately 9.6 million people died from various forms of cancer $(1,2)$. Pneumonia caused by influenza was responsible for an estimated 145,000 deaths (3).

With the advances in screening, diagnosis, and treatment of cancer in recent years, the survival rate of cancer patients has significantly improved (4); however, the morbidity and mortality of other non-cancer diseases such as stroke, cardiac disease, and Alzheimer's disease among cancer survivors remain increasing among the cancer survivors (5-8). It was reported that pneumonia was a major cause of death among long-term childhood cancer survivors (9). With age, cancer survivors admitted to the hospital due to pneumonia have an increased risk of dying from pneumonia (9). Among cancer patients, life-threatening pneumonia was mainly caused by the disease and its treatment comprising poor nutritional status and cytotoxic therapy (10-12). Moreover, higher doses of exercise, especially aerobic exercise has proven to be strongly associated with a lower risk of pneumonia mortality in a dose-dependent manner $(13,14)$.

Among patients with bone tumors, activities are restricted due to pain, distortion of bone and pathological fractures (15). The change of gait, deviation of appearance, and reductions in function, stability, and strength cause severe impairment of athletic ability of patients with malignant bone tumors, especially in those who underwent amputation (15-17). Furthermore, it was recognized that the most of child patients with primary malignant bone tumors usually suffer from malnutrition (18). These may increase the risk for fatal pneumonia $(10,11,13,14)$. To the best of our knowledge, there are few guidelines for the primary prevention and management of pneumonia in patients with malignant bone tumors. Therefore, the objectives of this study were to determine the risk of dying from pneumonia among patients with malignant bone tumors relative to that of the general population and to identify demographic and tumor-related characteristics and type of surgery associated with a higher risk of dying from pneumonia. We present the following article in accordance with the STROBE reporting checklist (available at https://dx.doi.org/10.21037/tcr-21-306).

\section{Methods}

\section{Data sources}

Patients diagnosed with malignant bone tumors between January 1st, 1973 and December 31st, 2016 were identified using the Surveillance, Epidemiology, and End Results (SEER) database, a publicly available, nationally representative, population-based cancer database established by the National Cancer Institute (19). The mortality data of the general US population collected by the National Center for Health Statistics spanning from 1969 to 2017 was also used as a comparison.

\section{Study population}

In this study, all patients with a diagnosis of malignant bone tumors were included. Patients were excluded if their cancer diagnosis were not microscopically confirmed and ages at the times of diagnosis and follow-up time were unknown (Figure 1). In the multivariate analysis, patients who did not have the definitive race, clinical stage, and information for surgery were also excluded.

\section{Study variables}

We extracted demographic characteristics from the SEER database, including age at diagnosis $(0-14,15-29,30-44$, $45-59$, and $60+$ years), sex (female, male), race (white, black, and other), and year of diagnosis (1973-1990, 1991-2000, 2001-2010, and 2011-2016). The tumor-related variables included the histological subtype, primary site (upper limb, lower limb, skull and face, vertebral column, thorax, pelvis, and others), and clinical stage of the bone tumors [localized (N0M0), regional (N+), distant (M1) and unknown (blank)]. The treatment variables comprised surgery (amputation, limb salvage, surgery, not otherwise specified, and none), chemotherapy (yes and none/unknown), and radiation (yes and none/unknown) for bone tumor. Survival time and cause of death were also available. All patients were divided into five subgroups according to the histological subtype of malignant bone tumors, including osteosarcoma, chondrosarcoma, Ewing sarcoma, chordoma, and others [International Classification of Disease for Oncology third 


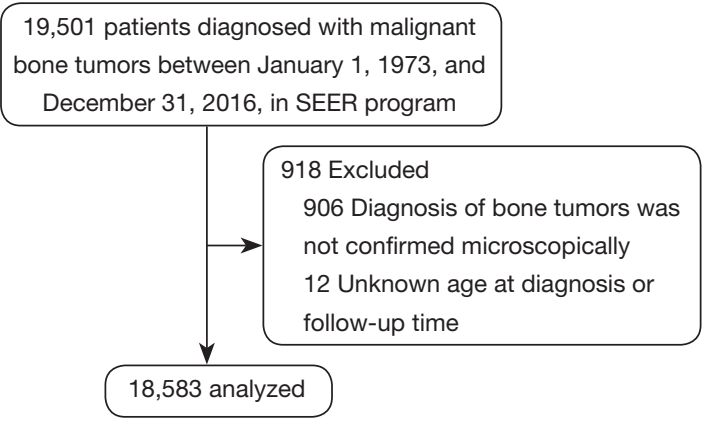

Figure 1 Flow diagram of patient selection within the SEER database between 1973 and 2016. SEER, Surveillance, Epidemiology, and End Results.

revision codes are listed in (Table 1)].

Patients with cause of death coded as "pneumonia and influenza (50120)" were considered to have died from pneumonia.

\section{Statistical analyses}

The number of deaths from pneumonia divided by personyears of survival was calculated as the mortality rate of pneumonia. Among cancer-patient subgroups stratified by different characteristics, the standardized mortality ratios (SMRs) and 95\% confidence intervals (95\% CIs) were calculated as described earlier (20), which provided the relative risk of fatal pneumonia for cancer patients compared with all general US population after adjusting the distribution difference of age, sex, and race among them. Five-year age ranges were used for standardization. In detail, the SMRs were estimated as the ratios of observed to expected number of deaths. The observed number of deaths represents the total number of deaths from fatal pneumonia among patients with bone tumors recorded during the study period; the expected number of deaths represents the number of individuals who died from fatal pneumonia in the general population with the same distribution of age at diagnosis, sex, race $(8,21)$. Other causes of non-cancer death other than pneumonia were regarded as competing risk events in the multivariate analysis. Therefore, we designed the Fine-Gray model to identify demographics, tumorrelated characteristics, and type of surgery associated with a higher risk of dying from pneumonia among patients with bone tumors. Interaction tests were further carried out to investigate the potential differential effect of amputation on relative risk of dying from pneumonia among patients with different histological subtype of bone tumors. Survival time recorded as 0 months in the SEER database was converted to one-half of a month according to accepted epidemiologic practices (20).

All statistical tests were 2 -sided, and $\mathrm{P}<0.05$ were also considered statistically significant. The analyses were performed using the SEER ${ }^{*}$ Stat software version 8.3.6 and the $\mathrm{R}$ version 3.51 statistical software.

\section{Ethical statement}

The study was conducted in accordance with the Declaration of Helsinki (as revised in 2013).

\section{Results}

Among 18,583 patients suffering from malignant bone tumors followed for 142,082 person-years, a total of 60 deaths due to pneumonia occurred, with a mortality rate of 42.23/100,000 person-years (Table 1). Patients with primary malignant bone tumors had a significantly higher risk of dying from pneumonia compared to the general US population with the same distribution of age, sex, and race (SMR $=2.79 ; 95 \%$ CI: 2.17-3.59). The interquartile range of survival time was from 1.33 to 11.33 years, with a median survival time of 4.25 years.

\section{Characteristics associated with an increased risk of dying from pneumonia}

\section{Demographics}

There were four deaths due to pneumonia observed among patients with malignant bone tumors whose age at diagnosis was less than 19 years (Figure 2). For these young cancer patients, the risk of dying from pneumonia was more than 10 -fold greater than that in the general population $(\mathrm{SMR}=10.07 ; 95 \% \mathrm{CI}: 5.24-19.36)$. The relative risk of fatal pneumonia gradually declined as patients with bone tumors grew older. The old patients whose age at diagnosis was more than 80 years old had the highest mortality rate of pneumonia among all age subgroups (1,312.19/100,000 person-years), but the risk of fatal pneumonia of those was equal to that of the general population (SMR $=1.80 ; 95 \%$ CI: 0.97-3.34). Blacks had a higher mortality rate of pneumonia despite the least number of deaths $(53.43 / 100,000$ person-years; SMR $=4.53 ; 95 \%$ CI: $2.16-9.51)$. 
Table 1 Deaths from pneumonia among patients with primary malignant bone tumors in the SEER database demographic and tumor characteristics

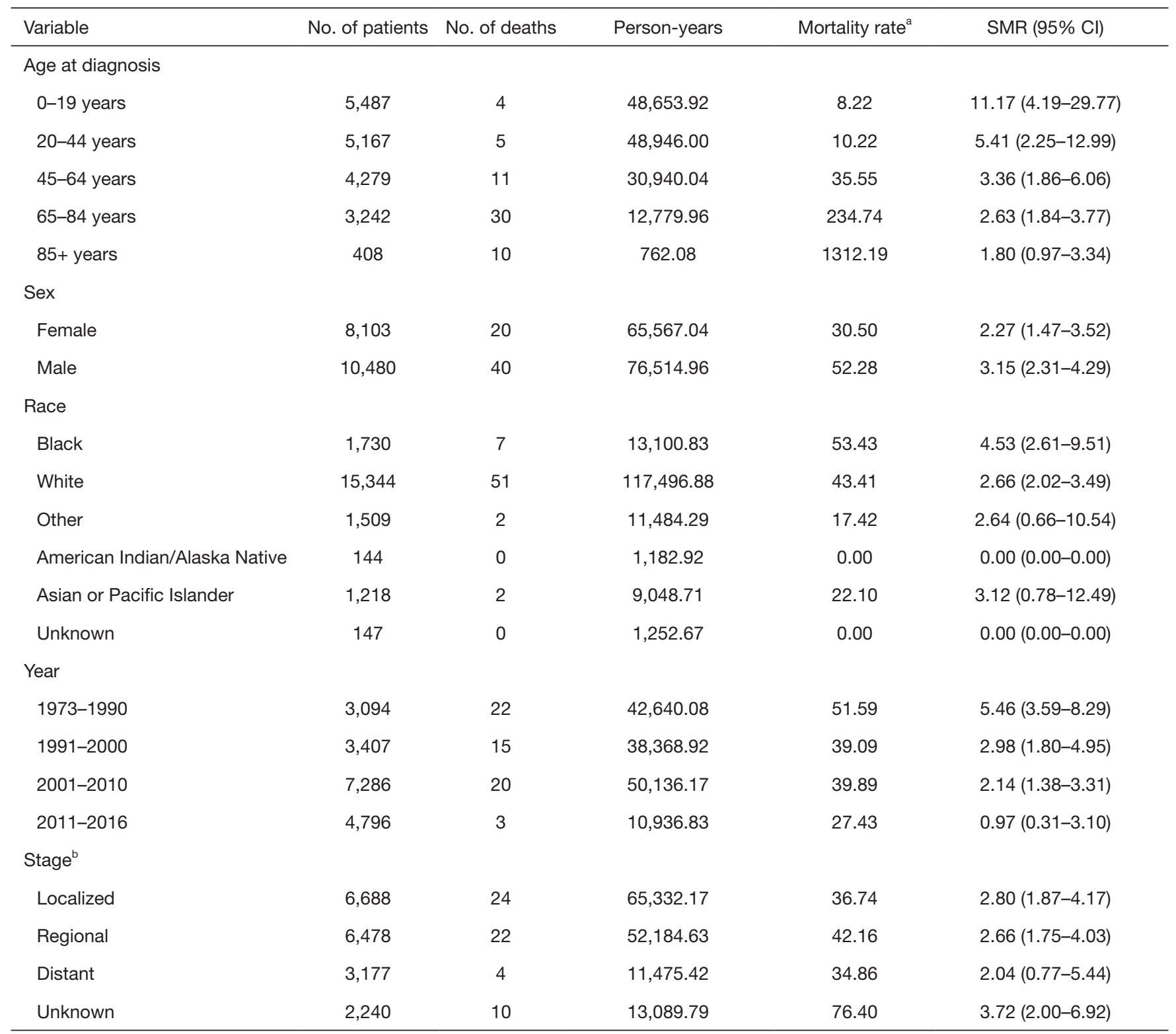

Table 1 (continued) 
Table 1 (continued)

\begin{tabular}{|c|c|c|c|c|c|}
\hline Variable & No. of patients & No. of deaths & Person-years & Mortality rate ${ }^{a}$ & SMR (95\% Cl) \\
\hline \multicolumn{6}{|l|}{ Primary site $^{c}$} \\
\hline Axial & 5,830 & 27 & $36,185.29$ & 74.62 & $3.03(2.08-4.42)$ \\
\hline Thorax & 1,440 & 10 & $11,477.54$ & 87.13 & 3.09 (1.66-5.74) \\
\hline Vertebral column & 1,299 & 7 & $7,960.29$ & 87.94 & 4.02 (1.92-8.43) \\
\hline Extremity & 9,699 & 24 & $84,791.21$ & 28.30 & $2.86(1.91-4.26)$ \\
\hline Upper limb & 2,298 & 5 & $19,832.83$ & 25.21 & $1.85(0.77-4.44)$ \\
\hline Lower limb & 7,401 & 19 & $64,958.38$ & 29.25 & $3.33(2.13-5.23)$ \\
\hline Others & 3,054 & 9 & $21,105.50$ & 42.64 & $2.14(1.11-4.11)$ \\
\hline \multicolumn{6}{|l|}{ Surgery } \\
\hline Yes & 14,162 & 40 & $119,267.42$ & 33.54 & $2.46(1.81-3.36)$ \\
\hline Amputation & 1,977 & 7 & $13,019.58$ & 53.77 & 3.29 (1.57-6.90) \\
\hline Limb salvage & 10,017 & 22 & $75,286.17$ & 29.22 & $1.92(1.26-2.92)$ \\
\hline Surgery, NOS & 2,168 & 11 & $30,961.67$ & 35.53 & $4.13(2.28-7.45)$ \\
\hline No & 3,547 & 11 & $15,317.17$ & 71.81 & $2.50(1.39-4.52)$ \\
\hline Unknown & 874 & 9 & $7,497.42$ & 120.04 & $10.38(5.40-19.95)$ \\
\hline \multicolumn{6}{|l|}{ Chemotherapy } \\
\hline All & 18,583 & 60 & $142,082.00$ & 42.23 & $2.79(2.17-3.59)$ \\
\hline
\end{tabular}

${ }^{\text {a }}$, per 100,000 person-years; ${ }^{\text {b }}$, localized: invasive but confined to the organ of origin; Regional: extension beyond the organ of origin; but no distant metastasis; Distant: distant metastasis; ${ }^{c}$, specific primary site code [the International Statistical Classification of Diseases and Related Health Problems, the tenth revision (ICD-O-10)]: Skull and face: C41.0, C41.1; Thorax: C41.3; Vertebral column: C41.2; Pelvis: C41.4; Upper limb: C40.0, C40.1; Lower limb: C40.2, C40.3; Others: C40.8, C40.9, C41.8, C41.9. SMR, standardized mortality ratio; CI, confidence interval.

\section{Tumor-related characteristics}

Patients with localized (36.74/100,000 person-years; SMR $=2.80 ; 95 \%$ CI: $1.87-4.17$ ) and distant bone tumors (34.86/100,000 person-years; SMR $=2.04$; $95 \%$ CI: 0.77 5.44) were equally likely to die from pneumonia. Besides, the relative risk of dying from pneumonia was highest in patients with malignant bone tumors of the vertebral column $(87.94 / 100,000$ person-years; SMR $=4.02 ; 95 \%$ CI: 1.92-8.43), followed by those with bone tumors of the lower limb (29.25/100,000 person-years; SMR $=3.33 ; 95 \%$ CI: $2.13-5.23)$, and the thorax $(87.13 / 100,000$ person-years; SMR $=3.09$; $95 \%$ CI: $1.66-5.74)$. 

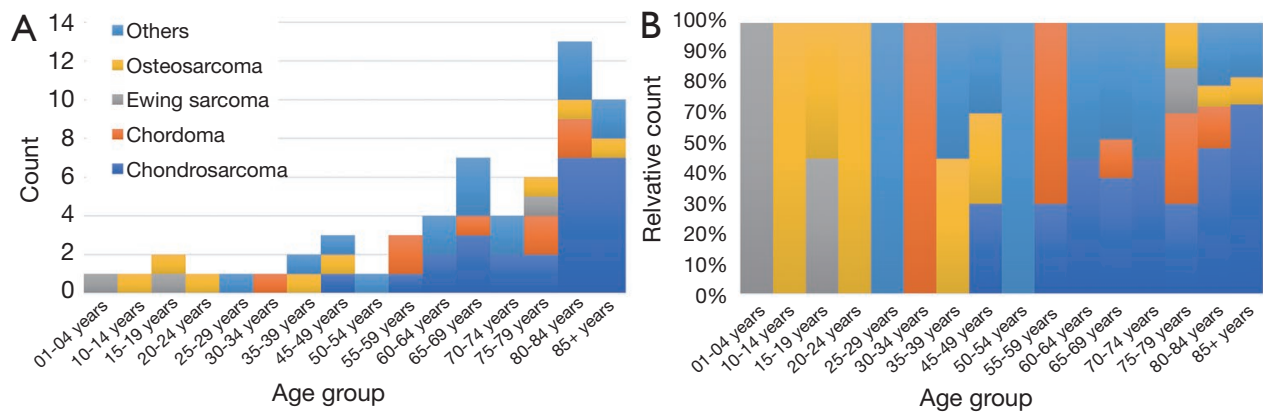

Figure 2 The y-axis represented the absolute or relative number of deaths due to pneumonia and the $\mathrm{x}$-axis represented the age group at diagnosis. The colors depicted the histological subtypes of malignant bone tumors. (A) Deaths from fatal pneumonia among cancer patients as a function of age group; (B) relative deaths from fatal pneumonia among cancer patients as a function of age group.

\section{Treatments for malignant bone tumors}

Patients who did not undergo surgery had a higher mortality rate of fatal pneumonia compared to patients who underwent surgery. The relative risk of fatal pneumonia was higher among patients who underwent amputation than those who underwent limb salvage or did not undergo surgery. Patients who underwent radiation (65.82/100,000 person-years; SMR $=3.53$; 95\% CI: $2.20-5.68)$ or did not undergo chemotherapy (60.34/100,000 person-years; SMR $=2.57$; $95 \%$ CI: $1.95-3.39$ ) for malignant bone tumors had a higher risk of fatal pneumonia.

\section{The risk factors for death from pneumonia based on the Fine-Gray model}

The Fine and Gray model indicated that older age at diagnosis and axial tumors were associated with the higher incidence of fatal pneumonia among patients with malignant bone tumors (Table 2). After controlling for potential confounders, amputation significantly increased the risk of dying from pneumonia compared with non-surgery and limb salvage [hazard ratio (HR) $=0.21 ; 95 \%$ CI: $0.06-0.77$; $\mathrm{P}=0.018]$ and (HR $=0.43 ; 95 \%$ CI: 0.19-0.93; $\mathrm{P}=0.033$ ).

\section{Pathological types of malignant bone tumors associated with a bigher risk of dying from pneumonia}

Among patients with chordoma, there were eight deaths due to pneumonia, accounting for $13 \%$ of all deaths due to pneumonia among patients with malignant bone tumors (Table 3). This was the highest mortality rate of pneumonia $(84.29 / 100,000$ person-years; SMR $=2.38 ; 95 \%$ CI: $1.19-4.76$ ), followed by patients with chondrosarcoma (53.83/100,000 person-years; SMR =2.34; 95\% CI: 1.58-3.46). Besides, patients with osteosarcoma (16.90/100,000 person-years; SMR $=2.99$; 95\% CI: 1.50 5.98) had approximately the same risk of fatal pneumonia as patients with Ewing sarcoma (16.11/100,000 personyears; SMR $=11.82 ; 95 \%$ CI: 3.81-36.64). The interaction test demonstrated that amputation increased the relative risk of fatal pneumonia among patients with osteosarcoma ( $\mathrm{SMR}=5.36$; 95\% CI: 1.34-21.43), but did not increased the relative risk among patients with other types of bone tumors.

\section{Time since diagnosis}

For all patients with malignant bone tumors, the mortality rate of fatal pneumonia was the highest within the first year after the cancer diagnosis $(89.47 / 100,000$ person-years; SMR $=2.92$; 95\% CI: 1.76-4.84), decreased gradually after one year, while the relative risk was the highest after ten years $(25.18 / 100,000$ person-years; SMR $=4.03 ; 95 \% \mathrm{CI}$ : 2.29-7.10). The highest relative suicide risks persisted over time in patients with osteosarcoma (Table 4). Figure 3 showed the cumulative incidence of death from pneumonia among cancer patients.

\section{Discussion}

Among patients with malignant bone tumors, surgical site infection is a common postoperative complication $(22,23)$, but few studies have paid attention to pulmonary infection. Using data from the SEER database, we first reported that the risk of dying from pneumonia among patients with primary malignant bone tumors was approximately three- 
Table 2 Fine-gray regression analysis for risk of dying from pneumonia among patients with malignant bone tumors

\begin{tabular}{|c|c|c|c|}
\hline Variable & $\mathrm{HR}$ & $95 \% \mathrm{Cl}$ & $P$ value \\
\hline Age at diagnosis & 8.53 & $(3.54,20.53)$ & $<0.001$ \\
\hline \multicolumn{4}{|l|}{ Sex } \\
\hline Female & - & - & Ref \\
\hline Male & 1.53 & $(0.73,3.20)$ & 0.260 \\
\hline \multicolumn{4}{|l|}{ Race } \\
\hline White & - & - & Ref \\
\hline Black & 0.99 & $(0.22,4.38)$ & 0.990 \\
\hline Other & 0.00 & $(0.00,0.00)$ & 0.000 \\
\hline \multicolumn{4}{|l|}{ Year of diagnosis } \\
\hline 1973-1990 & - & - & Ref \\
\hline $1991-2000$ & 0.85 & $(0.18,3.96)$ & 0.840 \\
\hline 2001-2010 & 0.76 & $(0.17,3.45)$ & 0.720 \\
\hline 2011-2016 & 0.33 & $(0.05,2.09)$ & 0.240 \\
\hline \multicolumn{4}{|l|}{ Location } \\
\hline Extremity & - & - & Ref \\
\hline Axial & 2.04 & $(1.03,4.05)$ & 0.041 \\
\hline Other & 1.32 & $(0.47,3.70)$ & 0.600 \\
\hline \multicolumn{4}{|l|}{ Stage } \\
\hline Distant & - & - & Ref \\
\hline Localized & 4.09 & $(0.88,18.89)$ & 0.072 \\
\hline Regional & 2.44 & $(0.54,11.02)$ & 0.240 \\
\hline \multicolumn{4}{|l|}{ Surgery } \\
\hline Amputation & - & - & Ref \\
\hline None & 0.21 & $(0.06,0.77)$ & 0.018 \\
\hline Limb salvage & 0.43 & $(0.19,0.93)$ & 0.033 \\
\hline \multicolumn{4}{|l|}{ Chemotherapy } \\
\hline No/unknown & - & - & Ref \\
\hline Yes & 0.58 & $(0.21,1.61)$ & 0.300 \\
\hline \multicolumn{4}{|l|}{ Radiation therapy } \\
\hline No/unknown & - & - & Ref \\
\hline Yes & 1.66 & $(0.67,4.12)$ & 0.270 \\
\hline
\end{tabular}

$\mathrm{HR}$, hazard ratio; $\mathrm{Cl}$, confidence interval; NOS, not otherwise specified. fold that of the general population. The risk factors of dying from pneumonia for these patients included lack of physical activities, prolonged bed rest, and malnutrition (12-14,18).

Our study demonstrated that the risk of dying from pneumonia among patients with bone tumors varied greatly by pathological subtypes of malignant bone tumors. In this study, patients with chordoma had the highest risk of dying from pneumonia, which may be associated with the common location of the chordoma. Chordoma is an extremely rare subtype of bone sarcoma and appears anywhere along the central neural axis from the skull base to the sacrum. Patients with chordomas usually present with tingling, numbness, weakness, and radiculopathies related to the level of spinal tumors (24-26), which could reduce exercise capacity and prolong the bed-rest time. Radical resection is the most basic treatment for chondroma, and the principles of surgical treatment for chordoma include more aggressive surgery, wider surgical margins, and elaborate reconstructive (27-29). Although the benefits of early mobilization after surgery have been well documented, the complexity of a spinal reconstructive procedure and poor overall physiologic condition in the early postoperative period may render early mobilization unfeasible (30-32). Additionally, among patients with bone tumors of the vertebral column, higher recurrence rates of spinal bone tumors increase the re-operation rates (33), thus further increasing the bed-rest time and incidence of in-hospital lung infections (34-36).

We found that amputation could significantly increase the risk of dying from pneumonia among patients with malignant bone tumors, mainly those associated with a lack of exercise. Patients often experience physical disability and motor dysfunction after amputation (15). In addition, the change of identity from being a healthy individual to becoming a disabled person has a negative effect on the mental aspects and social life of patients with bone tumors $(17,37)$. High levels of anxiety and depression have been observed in patients with bone tumors after remission $(38,39)$, which reduce patients' participation in sports and physical activity (15). To improve the exercise capacity of patients with amputation, clinicians could increase the utilization of sports prosthetic devices or popularize sports programs that do not require prosthetics such as wheelchair exercises or traditional Indian yoga among patients with 
Table 3 The risk of death from pneumonia by type of histology and surgery among patients with malignant bone tumors

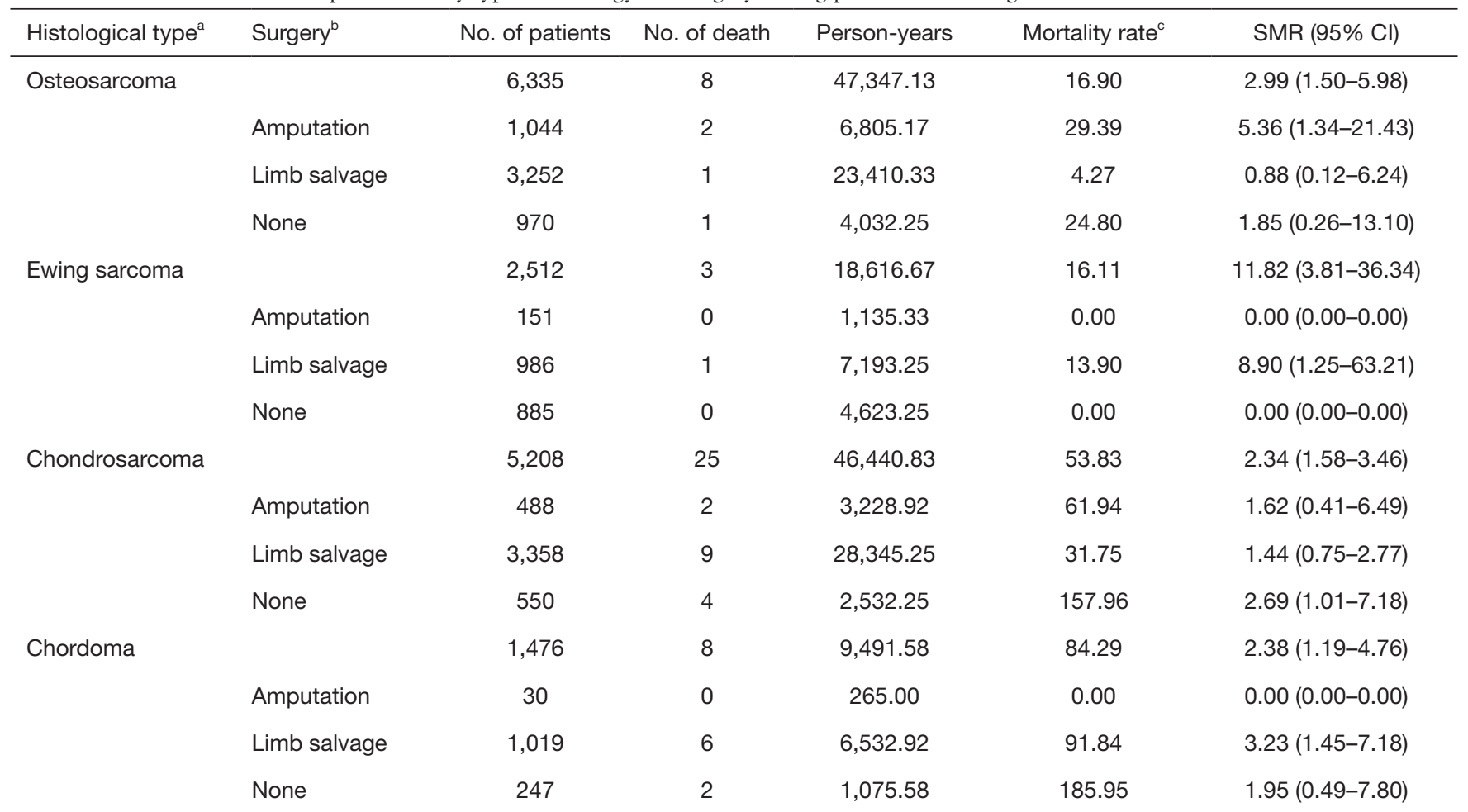

a, the histological codes (International Classification of Diseases for Oncology, 3rd Edition): Osteosarcoma: 9180/3-9200/3; Chondrosarcoma: 9220/3-9243/3; Ewing sarcoma: 9260/3; Chordoma: 9370/3-9372/3; Others: 8000/3-9150/3, 9250/3-9251/3, 9261/ $3-9365 / 3$ and $9380 / 3-9580 / 3$; ${ }^{b}$, these data about patients whose information of surgery was incomplete was not presented; ${ }^{\circ}$, per 100,000 person-years. SMR, standardized mortality ratio; CI, confidence interval; NOS, not otherwise specified.

bone tumors, which were deemed feasible $(15,40)$.

It was reported that almost one-third of deaths from pneumonia were among children younger than five years (1). Furthermore, although the number of people dying from pneumonia has decreased substantially over the past three decades, the number of deaths among people aged 70 years and older has increased (1). In an investigation of a long-term cancer survivor cohort in England, Smith et al. reported that childhood cancer survivors have a higher additional risk of hospitalization due to pneumonia compared with adolescent and young adult cancer survivors (9). We found that the young patients had a significantly higher risk of fatal pneumonia than the general population. Even though the patients whose age at diagnosis was more than 85 years had the highest mortality rate from pneumonia, this risk was equal to that of the general population. These meant that for young cancer patients, malignant bone tumors and related treatment play an important role in increased risk of fatal pneumonia, but for old patients, the age was the most predominant factor for the high mortality rate of pneumonia and the effects of tumors were negligible. Therefore, it was necessary to separate young and old patients as two distinct groups when considering the risk of dying from pneumonia among patients with malignant bone tumors.

We also found that the risk of dying from pneumonia was the highest within the first year after the initial diagnosis among patients with malignant bone tumors. Furthermore, patients who underwent amputation for bone tumors had a longer high-risk period than patients who did not undergo surgery, in which cancer patients had a higher incidence of fatal pneumonia compared with the general population. A possible hypothesis to explain this was that bone tumors and the related surgery resulted in a higher risk of fatal pneumonia shortly after diagnosis. With the improvement of the nutritional status and the recovery of exercise capacity among patients, the risk of pneumonia decreased gradually. However, the recovery of exercise capacity was difficult and required more time for patients who underwent amputation. These findings highlight that clinicians should have a 
Table 4 The incidence of fatal pneumonia by time since diagnosis

\begin{tabular}{|c|c|c|c|c|}
\hline Surgery & \multicolumn{4}{|c|}{ Time since diagnosis } \\
\hline \multicolumn{5}{|l|}{ All } \\
\hline No. of death & 15 & 21 & 12 & 12 \\
\hline Person-years & $16,765.92$ & $44,816.50$ & $34,106.83$ & $47,652.33$ \\
\hline SMR (95\% Cl) & $2.92(1.76-4.84)$ & $2.27(1.48-3.47)$ & $2.70(1.53-4.76)$ & $4.03(2.29-7.10)$ \\
\hline \multicolumn{5}{|l|}{ Osteosarcoma } \\
\hline No. of death & 3 & 7 & 3 & 4 \\
\hline Person-years & $5,705.46$ & $14,277.08$ & $10,845.50$ & $16,943.58$ \\
\hline \multicolumn{5}{|l|}{ Chondrosarcoma } \\
\hline No. of death & 4 & 5 & 2 & 3 \\
\hline Person-years & $4,797.08$ & $14,294.08$ & $11,800.92$ & $15,918.50$ \\
\hline Mortality rate & 83.38 & 34.98 & 16.95 & 18.85 \\
\hline SMR (95\% Cl) & $1.87(0.70-4.99)$ & $1.10(0.46-2.65)$ & $0.84(0.21-3.36)$ & $1.69(0.55-5.25)$ \\
\hline \multicolumn{5}{|l|}{ Ewing sarcoma } \\
\hline No. of death & 0 & 0 & 0 & 3 \\
\hline Person-years & $2,353.83$ & $5,958.67$ & $4,181.25$ & $6,302.08$ \\
\hline Mortality rate & 0.00 & 25.55 & 0.00 & 0.00 \\
\hline SMR (95\% Cl) & - & $0.57(0.08-4.06)$ & - & - \\
\hline
\end{tabular}

SMR, standardized mortality ratio; $\mathrm{Cl}$, confidence interval.

comprehensive understanding of the factors associated with a higher risk of dying from pneumonia, to mitigate the risk of dying from pneumonia among patients with bone tumors.

Our study has several limitations. First, influenza not only causes pneumonia but also has a negative effect on other systems, including the central nervous system and the cardiovascular system $(41,42)$. There is a possibility that the patients whose causes of death were coded as "pneumonia and influenza" did not die from pneumonia caused by influenza, but from complications caused by the influenza in the other systems. Second, the higher incidence of fatal pneumonia among patients with amputation is associated with lower dose of exercise, but we could not get quantified data about exercise from SEER database. Therefore, we could not directly evaluate the impact of exercise on the risk of fatal pneumonia among these patients.

\section{Conclusions}

We first reported that patients with malignant bone tumors 


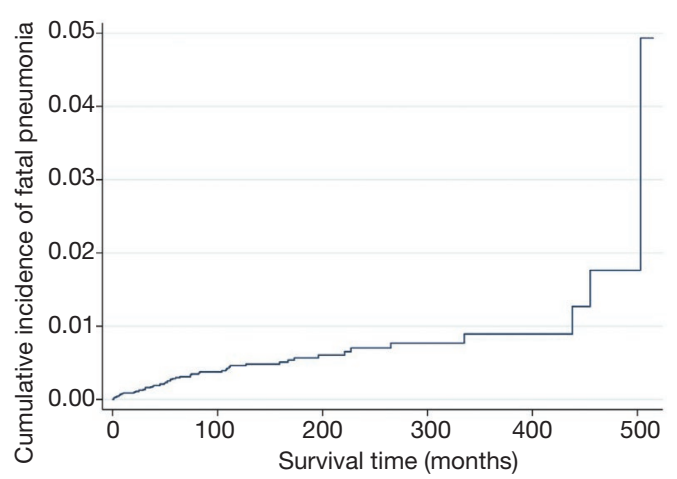

Figure 3 Cumulative incidence curve for death from pneumonia among patients with malignant bone tumors.

had a higher risk of dying from pneumonia compared to the general population. Surgery could decrease the incidence of fatal pneumonia, but amputation could increase the risk. Orthopedic surgeons cannot subjectively change the method of surgery for bone tumors. Therefore, we call for long-term clinical monitoring of the lung condition among these patients. This requires enhanced coordinated multidisciplinary cooperation between orthopedic and respiratory medicine teams.

\section{Acknowledgments}

We would like to thank EditorBar (https://www.editorbar. $\mathrm{com} /$ ) for his/her help in polishing our paper.

Funding: Wuhan Huanghe Talents Program, source of funds: Wuhan Talent Office, Fund number: HHYC201601. Department of science and technology of Hubei Province, Fund number: 2020BCB049. Fundamental Research Funds for the Central Universities, Fund number: HUST: 2021 yjsCXCY087.

\section{Footnote}

Reporting Checklist: The authors have completed the STROBE reporting checklist. Available at https://dx.doi. org/10.21037/tcr-21-306

Peer Review File: Available at https://dx.doi.org/10.21037/ tcr-21-306

Conflicts of Interest: All authors have completed the ICMJE uniform disclosure form (available at https://dx.doi. org/10.21037/tcr-21-306). The authors have no conflicts of interest to declare.

Ethical Statement: The authors are accountable for all aspects of the work in ensuring that questions related to the accuracy or integrity of any part of the work are appropriately investigated and resolved. The study was conducted in accordance with the Declaration of Helsinki (as revised in 2013). Institutional ethical approval and informed consent were waived.

Open Access Statement: This is an Open Access article distributed in accordance with the Creative Commons Attribution-NonCommercial-NoDerivs 4.0 International License (CC BY-NC-ND 4.0), which permits the noncommercial replication and distribution of the article with the strict proviso that no changes or edits are made and the original work is properly cited (including links to both the formal publication through the relevant DOI and the license). See: https://creativecommons.org/licenses/by-nc-nd/4.0/.

\section{References}

1. Our World in Data: Cancer. Available online: https:// ourworldindata.org/cancer

2. Bernadeta Dadonaite (2018) - "Pneumonia". Published online at OurWorldInData.org. Available online: https:// ourworldindata.org/pneumonia

3. GBD 2017 Influenza Collaborators. Mortality, morbidity, and hospitalisations due to influenza lower respiratory tract infections, 2017: an analysis for the Global Burden of Disease Study 2017. The Lancet Respiratory Medicine 2019;7:69-89.

4. Coleman MP, Gatta G, Verdecchia A, et al. EUROCARE-3 summary: cancer survival in Europe at the end of the 20th century. Ann Oncol 2003;14 Suppl 5:v128-49.

5. Zaorsky NG, Zhang Y, Tchelebi LT, et al. Stroke among cancer patients. Nat Commun. 2019;10:5172.

6. Sturgeon KM, Deng L, Bluethmann SM, et al. A population-based study of cardiovascular disease mortality risk in US cancer patients. Eur Heart J 2019;40:3889-97.

7. Mezencev R, Chernoff YO. Risk of Alzheimer's Disease in Cancer Patients: Analysis of Mortality Data from the US SEER Population-Based Registries. Cancers (Basel) 2020;12:796.

8. Misono S, Weiss NS, Fann JR, et al. Incidence of suicide in persons with cancer. J Clin Oncol 2008;26:4731-8. 
9. Smith L, Glaser AW, Peckham D, et al. Respiratory morbidity in young people surviving cancer: Populationbased study of hospital admissions, treatment-related risk factors and subsequent mortality. Int J Cancer 2019;145:20-8.

10. Wong JL, Evans SE. Bacterial Pneumonia in Patients with Cancer: Novel Risk Factors and Management. Clin Chest Med 2017;38:263-77.

11. Safdar A, Armstrong D. Infectious morbidity in critically ill patients with cancer. Crit Care Clin. 2001;17:531-viii.

12. Joffe L, Dwyer S, Glade Bender JL, et al. Nutritional status and clinical outcomes in pediatric patients with solid tumors : A systematic review of the literature. Semin Oncol 2019;46:48-56.

13. Williams PT. Dose-response relationship between exercise and respiratory disease mortality. Med Sci Sports Exerc 2014;46:711-7.

14. Teasell R, Dittmer DK. Complications of immobilization and bed rest. Part 2: Other complications. Can Fam Physician 1993;39:1440-2, 1445-6.

15. Bragaru M, Dekker R, Geertzen JH, et al. Amputees and sports: a systematic review. Sports Med 2011;41:721-40.

16. Fauske L, Bruland OS, Grov EK, et al. Cured of primary bone cancer, but at what cost: a qualitative study of functional impairment and lost opportunities. Sarcoma 2015;2015:484196.

17. Fauske L, Lorem G, Grov EK, et al. Changes in the body image of bone sarcoma survivors following surgical treatment--A qualitative study. J Surg Oncol 2016;113:229-34.

18. van Eys J. Malnutrition in children with cancer: incidence and consequence. Cancer 1979;43:2030-5.

19. Surveillance Epidemiology and End Results (SEER) Program: Overview of the SEER Program. Available online: https://seer.cancer.gov/

20. Koepsell TD, Weiss NS. Epidemiologic Methods: Studying the Occurrence of Illness. New York, NY: Oxford University Press, 2003.

21. Yang K, Zheng Y, Peng J, et al. Incidence of Death From Unintentional Injury Among Patients With Cancer in the United States. JAMA Netw Open 2020;3:e1921647.

22. Racano A, Pazionis T, Farrokhyar F, et al. High infection rate outcomes in long-bone tumor surgery with endoprosthetic reconstruction in adults: a systematic review. Clin Orthop Relat Res 2013;471:2017-27.

23. Miwa S, Shirai T, Yamamoto N, et al. Risk factors for surgical site infection after malignant bone tumor resection and reconstruction. BMC Cancer 2019;19:33.
24. Bergh P, Kindblom LG, Gunterberg B, et al. Prognostic factors in chordoma of the sacrum and mobile spine: a study of 39 patients. Cancer 2000;88:2122-34.

25. Fourney DR, Gokaslan ZL. Current management of sacral chordoma. Neurosurg Focus 2003;15:E9.

26. Walcott BP, Nahed BV, Mohyeldin A, et al. Chordoma: current concepts, management, and future directions. Lancet Oncol 2012;13:e69-76.

27. Hsieh PC, Xu R, Sciubba DM, et al. Long-term clinical outcomes following en bloc resections for sacral chordomas and chondrosarcomas: a series of twenty consecutive patients. Spine (Phila Pa 1976) 2009;34:2233-9.

28. Stacchiotti S, Casali PG, Lo Vullo S, et al. Chordoma of the mobile spine and sacrum: a retrospective analysis of a series of patients surgically treated at two referral centers. Ann Surg Oncol 2010;17:211-9.

29. Tzortzidis F, Elahi F, Wright D, et al. Patient outcome at long-term follow-up after aggressive microsurgical resection of cranial base chordomas. Neurosurgery 2006;59:230-7; discussion 230-7.

30. Marco RA, Stuckey RM, Holloway SP. Prolonged bed rest as adjuvant therapy after complex reconstructive spine surgery. Clin Orthop Relat Res 2012;470:1658-67.

31. McEvoy RD, Bradford DS. The management of burst fractures of the thoracic and lumbar spine. Experience in 53 patients. Spine (Phila Pa 1976) 1985;10:631-7.

32. Mumford J, Weinstein JN, Spratt KF, et al. Thoracolumbar burst fractures. The clinical efficacy and outcome of nonoperative management. Spine (Phila Pa 1976) 1993;18:955-70.

33. Yin $\mathrm{H}$, Zhou W, Meng J, et al. Prognostic factors of patients with spinal chondrosarcoma: a retrospective analysis of 98 consecutive patients in a single center. Ann Surg Oncol 2014;21:3572-8.

34. Nursing in Pneumonia. Am J Nurs 2019;119:69.

35. Geissen NM, Medairos R, Davila E, et al. Number of Ribs Resected is Associated with Respiratory Complications Following Lobectomy with en bloc Chest Wall Resection. Lung 2016;194:619-24.

36. Pakzad H, Roffey DM, Knight H, et al. Delay in operative stabilization of spine fractures in multitrauma patients without neurologic injuries: effects on outcomes. Can J Surg 2011;54:270-6.

37. Siracuse BL, Gorgy G, Ruskin J, et al. What is the Incidence of Suicide in Patients with Bone and Soft Tissue Cancer? : Suicide and Sarcoma. Clin Orthop Relat Res 2017;475:1439-45.

38. Paredes T, Canavarro MC, Simões MR. Anxiety and 
depression in sarcoma patients: emotional adjustment and its determinants in the different phases of disease. Eur J Oncol Nurs 2011;15:73-9.

39. Wiener L, Battles H, Bernstein D, et al. Persistent psychological distress in long-term survivors of pediatric sarcoma: the experience at a single institution. Psychooncology 2006;15:898-910.

40. Song Y, Ren F, Sun D, et al. Benefits of Exercise on

Cite this article as: Yu K, Chen Y, Tian Y, Kang H, Song K, Dong Y, Peng R, Li F. Characteristics, incidence, and risk factors for death from fatal pneumonia among patients with primary malignant bone tumors: a SEER-based observational study. Transl Cancer Res 2021;10(8):3659-3670. doi: 10.21037/tcr21-306
Influenza or Pneumonia in Older Adults: A Systematic Review. Int J Environ Res Public Health 2020;17:2655.

41. Clerkin KJ, Fried JA, Raikhelkar J, et al. COVID-19 and Cardiovascular Disease. Circulation 2020;141:1648-55.

42. Mao L, Jin H, Wang M, et al. Neurologic Manifestations of Hospitalized Patients With Coronavirus Disease 2019 in Wuhan, China. JAMA Neurol 2020;77:683-90. 